\title{
TENS in Radiation Induced Xerostomia - Case Reports
}

\author{
Vijaykumar Bokkasam ${ }^{1}$, Yamini Puchalapalli², Sameeulla Shaik ${ }^{3}$, S.V. Suman ${ }^{4}$, Sai Praveen ${ }^{5}$, Yendluru Mercy $^{6}$, \\ Naga Jyothi Peta7, Sai Jyotsna Siddavaram ${ }^{8}$, Nikitha Reddy Veluru ${ }^{9}$ \\ ${ }^{1}$ Professor and HOD, Department of Oral Medicine and Radiology, ${ }^{2}$ Post Graduate, Department of Oral Medicine and \\ Radiology, ${ }^{3}$ Reader, Department of Oral Medicine and Radiology, ${ }^{4}$ Professor, Department of Oral Medicine and Radiology, \\ ${ }^{5}$ Senior Lecturer, Department of Oral Medicine and Radiology, ${ }^{6}$ Senior Lecturer, Department of Oral Medicine and Radiology, \\ ${ }^{7}$ Post Graduate, Department of Oral Medicine and Radiology, ${ }^{8}$ Post Graduate, Department of Oral Medicine and Radiology, \\ CKS Theja Dental College, Renigunta Road, Tirupathi, ' ${ }^{9}$ ral Medicine and Radiology Consultant and Independent Academic, \\ Nellore, Andhra Pradesh, India
}

Corresponding author: Puchalapalli Yamini, Post Graduate In Department of Oral Medicine and Radiology, India

DOI: http://dx.doi.org/10.21276/ijcmsr.2020.5.2.12

How to cite this article: Vijaykumar Bokkasam, Yamini Puchalapalli, Sameeulla Shaik, S.V. Suman, Sai Praveen, Yendluru Mercy, Naga Jyothi Peta, Sai Jyotsna Siddavaram, Nikitha Reddy Veluru. TENS in radiation induced xerostomia - case reports. International Journal of Contemporary Medicine Surgery and Radiology. 2020;5(2):B52-B54.

\section{A B S T R A C T}

Introduction: Xerostomia refers to a subjective sensation of dry mouth which is frequently associated with salivary gland hypofunction. Radiation induced xerostomia is a sequel of irradiation of head and neck cancers. Along with palliative treatment, electrostimulation plays a pivotal role in treatment of xerostomia. Transcutaneous electrical nerve stimulation (TENS) is a well-known, non invasive, safe physical therapy which stimulates salivary glands to increase whole salivary flow rate in patients undergoing radiation therapy.

Case report: In this case report we evaluated the effectiveness of TENS therapy in stimulating salivation in radiation-induced xerostomia patients.

Conclusion: TENS is a non-invasive procedure and the results are encouraging with minimal side effects, TENS can be used as a palliative treatment modality for post-radiation-induced xerostomia.

Keywords: Transcutaneous Electrical Nerve Stimulation, Radiation Induced Xerostomia, Irradiation.

\section{INTRODUCTION}

Saliva is a physiological fluid of the oral cavity. It is essential for host defense, cleansing of the oral cavity, and maintains neutral $\mathrm{PH}^{\mathrm{H}}$ to prevent tooth demineralization. Saliva provides lubrication and moisture to facilitate speech and taste. Major salivary glands contribute to $90 \%$ of saliva production. ${ }^{1}$

Radiotherapy is a therapeutic modality used to treat cancer. The success of the treatment of cancer with radiotherapy and chemotherapy is based on the absence of recurrence and survival of the patient, but actual success also lies in the overall well being of the patient after radiotherapy. So we showed a look at the improved quality of life even after radiotherapy for which post-radiation effects should be addressed well. Radiotherapy for head and neck malignancies leads to functional impairment of salivary glands; mostly parotid gland was affected that results in reduced salivary flow. More than $53 \mathrm{~Gy}$ of radiation to salivary gland causes irreversible damage, but to treat head and neck malignancies, 50-70 Gy of radiation is needed. ${ }^{2}$

Xerostomia results in demineralization of enamel, increased plaque accumulation, mucositis, burning mouth, absent or reduced salivary pooling, dryness of mouth, halitosis, difficulty in talking, chewing, and swallowing. ${ }^{3}$
Palliative treatments for xerostomia like ice-chips, saliva substitutes, citric acid, sugar-free chewing gums, systemic sialogogues like pilocarpine and cevimeline works well for some patients, but all have met with limited success. ${ }^{1}$

Transcutaneous electrical nerve stimulation (TENS) is a non-invasive and nonpharmacological method for electrical stimulation of the nerves through electrodes. It is widely used to relieve various types of medical conditions such as postoperative pain, low back pain, psychosomatic and arthritic pain, neurogenic pain, and visceral pain. ${ }^{4}$ In dentistry, TENS used to treat chronic pain in maxillofacial regions like myofascial pain, atypical neuralgia, TMJ pains, and to increase the salivary flow rate in xerostomia. ${ }^{5,6}$

\section{CASE REPORT 1}

A 52 years female patient visited the department of oral medicine and radiology with a chief complaint of dry mouth, burning sensation, dysphagia during swallowing of solid foods, and reduced mouth opening since 11 months. She had undergone radiotherapy for carcinoma of buccal mucosa for 6weeks with a total dose of $66 \mathrm{~Gy}$ in 33 fractions along with three cycles of cisplatin chemotherapy 11 months back. Clinical examination revealed extraoral pigmentation over the lower third of the face. The maximum inter incisal 
distance was $9 \mathrm{~mm}$ with restricted tongue protrusion. She had reduced salivary production with dry and sticky buccal mucosa. Based on history and clinical examination diagnosed as radiation-induced xerostomia and radiation-induced fibrosis, she was treated tens therapy, and mouth opening exercises were suggested.

TENS unit used was bioresearch quadra TENS in Single pulse mode with a Pulse width of $60 \mu$ sec and frequency of $600 \mathrm{~Hz}$. Electrodes were placed externally on the skin in the preauricular region to stimulate the parotid gland and in the submandibular region to stimulate the submandibular gland with the TENS unit in off position (fig.1). Unstimulated whole saliva collected in a graduated test tube for 5 minutes before the TENS therapy was $0.8 \mathrm{ml} / 5 \mathrm{~min}$ (fig.2). TENS unit was activated for 10 minutes and stimulated saliva was collected for 5 minutes in a separate graduated test tube. TENS therapy was given twice a week for three weeks. In the last session of TENS therapy, unstimulated saliva was increased to $1.2 \mathrm{ml} / 5 \mathrm{~min}$ and stimulated saliva was increased to $1.5 \mathrm{ml} / 5 \mathrm{~min}$ (fig. 3 ).

\section{CASE REPORT 2}

A 58 years male patient visited the department of oral medicine and radiology with a chief complaint of dry mouth, burning sensation, dysphagia during swallowing of solid foods, and reduced mouth opening and decayed teeth for seven years. He had undergone radiotherapy for carcinoma of tongue seven years back. Clinical examination revealed Maximum inter incisal distance is $1.5 \mathrm{~cm}$ with restricted tongue protrusion. He had reduced salivary production with dry and sticky buccal mucosa and all the teeth are grossly decayed. Based on history and clinical examination diagnosed as radiation-induced xerostomia and radiation-induced fibrosis and caries. He was treated with TENS similarly as in case 1 , and mouth opening exercises were suggested.

Unstimulated whole saliva collected in a graduated test tube for 5 minutes before the TENS therapy was zero. TENS unit was activated for 10 minutes and stimulated saliva was collected for 5 minutes in a separate graduated test tube. TENS therapy was given twice a week for three weeks. In the last session of TENS therapy, unstimulated saliva was increased to $0.3 \mathrm{ml} / 5 \mathrm{~min}$ and stimulated saliva was increased to $0.7 \mathrm{ml} / 5 \mathrm{~min}$.

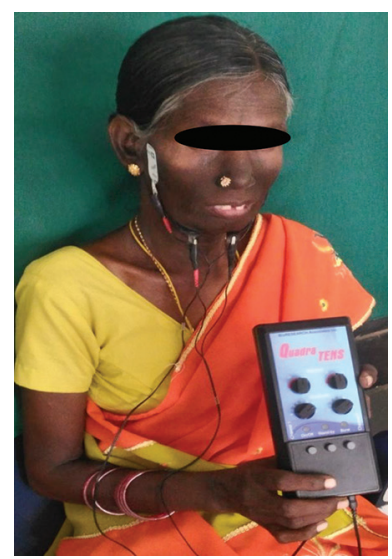

Figure-1: Electrodes are placed over parotid and submandibular gland

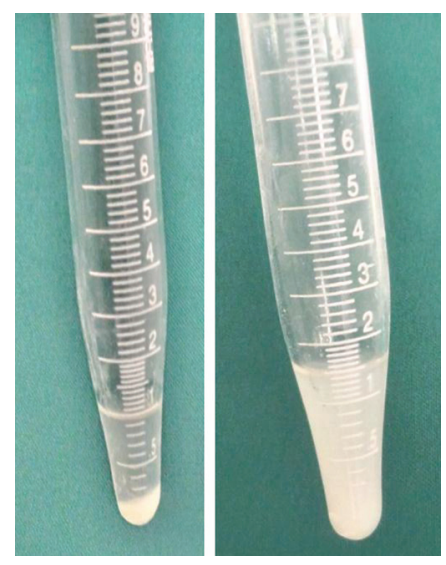

Figure 2 \& 3: Unstimulated and stimulated saliva collected in a graduated test tube

\section{DISCUSSION}

TENS is a safe, non-invasive, drug-free method for nerve stimulation. The unit used is Quadra TENS model QT - 42 (Bioresearch Associates Inc.) that consists of a central control unit, electrode pads, and lead wire to connect electrodes to the control panel. Quadra TENS has electrodes to stimulate four areas at a time; in our case, we stimulated parotid and submandibular glands bilaterally. It works with a $9 \mathrm{~V}$ battery.

\section{Mechanism of action of salivary stimulation:}

Tens directly stimulate the auriculotemporal nerve that supplies secretomotor innervations to the parotid gland. Salivation is controlled by both sympathetic and parasympathetic efferent nerves. Sympathetic stimulation produces sparse, viscous saliva for which higher frequencies and longer pulse duration are required. On the other hand, parasympathetic stimulation of the salivary glands produces copious amounts of watery saliva at lower frequencies. This voluminous serous saliva was useful for the management of xerostomia. $^{7,8}$

Singh D et al. ${ }^{1}$ conducted a study to evaluate the efficacy of TENS therapy in healthy adult subjects, which showed there is a significant increase in salivary flow. In the first case, the unstimulated salivary flow rate was $0.8 \mathrm{ml} / 5 \mathrm{~min}$, which was increased to $1.2 \mathrm{ml} / 5 \mathrm{~min}$ after five sessions of TENS stimulation. In the second case, the unstimulated salivary flow rate was zero, which was increased to $0.5 \mathrm{ml} / 5 \mathrm{~min}$.

Our cases showed increased salivary flow rate after stimulation by TENS therapy compared to the unstimulated salivary flow which was in agreement with the study by Ojha S et al. ${ }^{4}$ in which 24 out of 30 patients who underwent radiotherapy for oral cancer demonstrated a significant increase in the salivary flow. Lakshman AR et al. ${ }^{7}$ conducted a study on 30 patients undergoing radiotherapy for head and neck cancer and ten healthy participants and concluded that TENS therapy was found to be effective when used along the full course of radiation therapy as a concurrent treatment modality and in healthy individuals. A Vijayan et al. ${ }^{9}$ had stimulated parotid gland with TENS at a frequency of $50 \mathrm{HZ}$ on 30 patients who submitted to radiotherapy for oropharyngeal cancer and found an increase in the salivary rate after the TENS was $0.06 \mathrm{ml} / \mathrm{minute}$ in the present case stimulated salivary flow was increased to $0.3 \mathrm{ml} / \mathrm{minute}$. 
In Dalbem paim et al. Study ${ }^{10}$, TENS stimulated the parotid gland in patients who received radiotherapy 90 days prior to the study, at a frequency of $50 \mathrm{HZ}$ twice a week for four weeks, up to 8 sessions, and showed a progressive increase in stimulated salivary flow from the third session until the end of the treatment and that remained unchanged until six months after treatment.

The only side effect we found twitching of facial musculature, which was minimal and transient and ceased on reducing the amplitude or after TENS unit was switched off.

TENS was effective in improving hyposalivation, and the results are promising to improve the quality of life in patients receiving radiotherapy for head and neck cancers.

\section{CONCLUSION}

As TENS is a non-invasive procedure, and the results are encouraging with minimal side effects, TENS can be used as a palliative treatment modality for post-radiation-induced xerostomia.

\section{REFERENCES}

1. Singh D, Agrawal S, Shashikanth MC, Misra N. The effects of transcutaneous electric nerve stimulation (TENS) on salivary flow: A study. J Indian Acad Oral Med Radiol 2015;27(1):16-9.

2. Pinna R, Campus G, Cumbo E, Mura I, Milia E. Xerostomia induced by radiotherapy: an overview of the physiopathology, clinical evidence, and management of the oral damage. Ther Clin Risk Manag. 2015;11(3):171-188.

3. Sultana N \& Sham E. Xerostomia: An overview. Int. J. Dent. Clin. 2011:3(2):58-61.

4. Ojha S, Bhovi TV, Jaju PP, Gupta M, Singh N, Shrivastava K. Effectiveness of transcutaneous electrical nerve stimulation on saliva production in post-radiated oral cancer patients. J Indian Acad Oral Med Radiol 2016;28(7):246-51.

5. Kasat V, Gupta A, Ladda R, Kathariya M, Saluja H, Farooqui AA. Transcutaneous electric nerve stimulation (TENS) in dentistry- A review. J Clin Exp Dent. 2014;6(5):e562-8.

6. Richard R. Black. Use of transcutaneous electrical nerve stimulation in dentistry. JADA, 1986;113(2):649-52.

7. Lakshman AR, Babu GS, Rao S. Evaluation of the effect of transcutaneous electrical nerve stimulation on salivary flow rate in radiation-induced xerostomia patients: A pilot study. J Can Res Ther 2015;11(6):229233.

8. Steller M, Chou L, Daniels TE. Electrical stimulation of salivary flow in patients with Sjögren's syndrome. J Dent Res 1988;67(4):1334-7.

9. Vijayan A., Asha M.L., Babu S., Chakraborty S. Prospective Phase II Study of the Efficacy of Transcutaneous Electrical Nerve Stimulation in Postradiation Patients. Clinical Oncology .2014;26(3):74347.

10. Dalbem Paim É, Costa Batista Berbert M, Gonzales Zanella V, Beatris Martins V, Edler Macagnan F. Effects of transcutaneous electrical nerve stimulation on the salivary flow of patients with hyposalivation induced by radiotherapy in the head and neck region-A randomized clinical trial. J Oral Rehabil. 2019;:1-9.

Source of Support: Nil; Conflict of Interest: None

Submitted: 23-03-2020; Accepted: 06-05-2020; Published online: 02-06-2020 